\title{
Radiothérapie hypofractionnée des cancers du sein chez la femme âgée: état des lieux et
} perspectives.

E. Rivin del Campo ${ }^{1}$, S. Rivera ${ }^{2}$,

${ }^{1}$ Service d'Oncologie Radiothérapie, Hôpital Tenon, Hôpitaux Universitaires Est Parisien, AP HP, 4 rue de la Chine, Paris, France ; Université Paris Sorbonne, Paris, France

${ }^{2}$ Département de Radiothérapie, Gustave Roussy Cancer Campus, 114 rue Edouard Vaillant, Villejuif, France ; Université Paris-Sud, Paris, France

Titre raccourci: Hypofractionnement et cancer du sein.

Auteur correspondant: Eleonor Rivin del Campo. Tél.: (33)612353378. Fax : (33)156016599 eleonorrivin@gmail.com

Service d'Oncologie Radiothérapie, Hôpital Tenon, 4 rue de la Chine, 75020, Paris, France.

Conflit d'intérêt : aucun. 


\section{RESUME}

La radiothérapie adjuvante est un élément clé du traitement des cancers du sein traités par chirurgie conservatrice. Elle apporte un bénéfice en contrôle local mais aussi en survie spécifique et globale. Il est donc crucial de délivrer de manière optimale ce traitement y compris chez les sujets âgés. Ces dernières années, plusieurs schémas de radiothérapie hypofractionnée ont démontré leur non infériorité par rapport au schéma standard normofractionné permettant une réduction du nombre total de séances et de la lourdeur du traitement de radiothérapie. L'objectif de cette revue de la littérature est de faire un état des lieux des indications validées de la radiothérapie hypofractionnée dans le traitement des cancers du sein en particulier chez la femme âgée et de présenter les perspectives de développement de ces schémas.

Mots-clés: Cancer du sein, hypofractionnement, radiothérapie, sujet âgé

\section{ABSTRACT}

Adjuvant radiotherapy is the pillar of breast conserving surgery. Its omission is related with a higher risk of local recurrence and of breast cancer related or non-related mortality. It is of paramount importance to guarantee this treatment is fully administered to all patients after breast conserving surgery, including the elderly. In the last three decades several hypofractionated treatment schemes have shown their non-inferiority, allowing for treatment 
time reduction facilitating adhesion to treatment and reducing radiotherapy burden. This review focuses on the schemes and indications which have been validated by large phase 3 trials and those which are still under evaluation.

Key words: Breast cancer, hypofractionation, radiotherapy, elderly.

\section{INTRODUCTION}

La radiothérapie adjuvante reste indispensable dans le traitement des cancers du sein infiltrant de stade précoce. Cette indication formelle repose sur les données de deux métaanalyses de l'Early Breast Cancer Trialists' Collaborative Group (EBCTCG). La première métaanalyse a évalué l'impact de la radiothérapie adjuvante après mastectomie et curage axillaire, alors que la deuxième a évalué ce traitement chez les patientes ayant eu une chirurgie conservatrice $(1,2)$.

De 1960 à 1980, 8.135 patientes, recevant ou non une radiothérapie en fractionnement conventionnel (1,8-2 Gy/ fraction) sur la paroi et les aires ganglionnaires locorégionales ont été randomisées dans 22 essais post-mastectomie et intervention axillaire. Ces patientes ont fait l'objet de la première méta-analyse (1). Parmi ces patientes, 3.887 ont eu un curage axillaire et toutes celles traitées par radiothérapie ont reçu une radiothérapie sur la paroi, les aires sus claviculaires et/ou axillaires et de la chaîne mammaire interne. Dans ce groupe de patientes, avec un suivi médian de 9,4 ans pour les récidives et de 20 ans pour la mortalité, le traitement par radiothérapie n’a pas montré de bénéfice en terme de réduction de récidive, ni de réduction de la mortalité, chez les patientes sans atteinte ganglionnaire (pNO). En revanche, 
chez les patientes avec atteinte ganglionnaire $(\mathrm{pN}+)$ cette méta-analyse a montré une réduction absolue du risque de récidive locorégionale (- 18\%), de récidive (toutes récidives confondues) (10,6\%) et de la mortalité liée au cancer du sein (-4\%) grâce à la radiothérapie adjuvante. Ce bénéfice chez les patientes $\mathrm{pN}+$ est observé pour les patientes avec 1 à 3 ganglions positifs ainsi que pour celles avec plus de 4 ganglions atteints. L'analyse par tranche d'âge a montré que ces résultats sont valables indépendamment de l'âge (3).

La deuxième méta-analyse a été réalisée sur 10801 patientes incluses dans 17 essais randomisant l'administration ou l'omission de la radiothérapie après une chirurgie mammaire conservatrice. Elle a montré un bénéfice en faveur de la radiothérapie pour toutes les tranches d'âge, avec globalement une réduction absolue du risque de récidive (locale ou à distance) à 10 ans de $14,7 \%$, une réduction absolue du risque de mortalité par cancer du sein à 15 ans de 3,8\% et de la mortalité globale à 15 ans de $3 \%$.

Ces données établissent avec un niveau de preuve IA I'indication de radiothérapie adjuvante chez les patientes mastectomisées pN+ (radiothérapie pariétale et ganglionnaire) et chez les patientes traitées par chirurgie conservatrice (sein seul au minimum), en confirmant le bénéfice en contrôle local, en survie spécifique et en survie globale indépendamment de l'âge de la patiente (1-3). L'essai de phase III de Bartelink et al. randomisant la réalisation d'une surimpression du lit opératoire chez 2657 patientes a démontré, une réduction significative de l'incidence cumulée à 20 ans des récidives locales (16,4\% versus $12 \%$ respectivement dans les bras sans et avec surimpression) (4). Mais, l'amplitude de ce bénéfice diminue avec l'âge avec une réduction absolue de l'incidence cumulée à 20 ans des récidives locales de 11,6\% chez les moins de 40 ans, de 5,9\% chez les 41 - 50 ans et d'environ 3\% à partir de 51 ans (4). 
Le traitement standard délivrant 50 Gy en 25 fractions sur le sein ou la paroi +/- les aires ganglionnaires +/- une surimpression du lit tumoral de 10 à 16 Gy en fractionnement conventionnel est relativement long et contraignant, en particulier pour les patientes âgées et/ou avec des trajets importants. En 2000, dans une étude aux Etats-Unis, Athas et al ont montré que jusqu'à 33\% des femmes traitées par chirurgie conservatrice n’ont pas reçu la radiothérapie adjuvante qu'elles auraient dû avoir, principalement du fait de l'âge supérieur à 70 ans et de la distance au centre de radiothérapie (5). Différents schémas hypofractionnés +/accélérés ont été testés pour répondre à cette problématique. Les données de 4 grands essais de phase III de non-infériorité, ont montré, avec un niveau de preuve IA, que la radiothérapie hypofractionnée du sein n'est pas inférieure à la radiothérapie normofractionnée tant en terme d'efficacité que de toxicité (6-8). Ainsi, depuis 2011, la société américaine de radiothérapie (ASTRO) a publié des recommandations d'utilisation des schémas hypofractionnés, chez les patientes d'au moins 50 ans, ayant un cancer du sein pT1-2, pN0, sans traitement par chimiothérapie adjuvante, sans indication de surimpression du lit tumoral (9). Depuis, plusieurs recommandations nationales et internationales ont également suivies (10-16). Au Royaume Uni, comme dans de nombreux pays, 40 Gy en 15 fractions est devenu le traitement standard d'irradiation mammaire voir pariétale, avec ou sans irradiation ganglionnaire (17).

\section{Chapitre 1 : L'hypofractionnement dans les cancers du sein infiltrants : une efficacité} démontrée. 
L'essai randomisé de phase III anglais, START-Pilot, a étudié deux schémas hypofractionnés (42,9 Gy et 39 Gy en 13 fractions sur 5 semaines) en comparaison avec un bras contrôle (50Gy en 25 fractions), en incluant 1410 patientes entre 1986 et 1998 (7). Ces schémas, utilisés chez des patientes ayant eu une chirurgie conservatrice, avec un suivi médian de 9,7 ans, ont montré un risque de récidive homolatérale de $12,1 \%$ pour le bras contrôle, 9,6\% pour le bras délivrant 42,9 Gy et $14,8 \%$ pour le bras délivrant 39 Gy. II n'a pas été mis en évidence de différence statistiquement significative entre les bras hypofractionnés et le bras standard. En revanche, il a été montré une différence significative des deux bras expérimentaux entre eux $(p=0,027)$ en faveur du bras à 42,9 Gy (7). Dans cet essai pilote le rapport $\alpha / \beta$ du cancer du sein a été estimé à 4 Gy (IC 95\% 1-7,8) tout comme celui pour la réaction tardive des tissus sains (7). Ceci signifie que le cancer du sein est probablement aussi sensible à la variation de dose par fraction que les tissus sains. Par conséquent, il serait possible de délivrer des doses par fractions plus importantes sans compromettre l'efficacité ou le risque de toxicité voir même en améliorant les $\operatorname{deux}(7)$.

Suite à cet essai pilote bi centrique, en 1999, deux autres essais anglais multicentriques ont été réalisés, les essais START-A et START-B (8). L'essai START-A a comparé 50 Gy en 25 fractions avec deux bras expérimentaux délivrant 41,6 Gy et 39 Gy en 13 fractions sur 5 semaines, chez des patientes pT1-3a, pN0-1, M0, dont $85 \%$ ont reçu une chirurgie conservatrice. Avec 2.236 patientes incluses, et un suivi médian de 9,3 ans, il n’a pas été montré de différence significative sur le taux de récidive locorégionale à 10 ans. Aucune différence significative n'a été observée entre les bras pour la survie sans maladie ni la survie globale. L'essai START-B, avec 2215 patientes pT1-3a, pN0-1, M0, d'âge médian 57 ans (23 à 86) 
a comparé un bras contrôle (50 Gy en 25 fractions) à un bras expérimental combinant un hypofractionnement modéré de 2,67 Gy par fraction et une accélération du traitement, administré sur 3 semaines au lieu de 5 (40 Gy en 15 fractions). La majorité des patientes (92\%) ont eu un traitement chirurgical conservateur. Avec un suivi médian de 9,9 ans, il n'a pas été retrouvé de différence significative sur le taux de récidive locoregionale à 10 ans entre les deux bras. En revanche, des taux de récidive à distance (HR 0,74, IC 95\% 0,59-0,94), de survie sans maladie (HR 0,79, IC 95\% 0,65-0,97; $p=0,022$ ) et de survie globale significativement meilleurs ( $p=0,042)$ ont été rapportés dans le bras expérimental (8).

L' essai multicentrique canadien a comparé le traitement standard (50 Gy en 25 fractions) à un traitement hypofractionné accéléré délivrant 42,5 Gy en 16 fractions, sur 22 jours (6). Entre 1993 et 1996, 1.234 patientes avec un cancer du sein infiltrant pT1-2, pN0, M0 ont été incluses. La population de cet essai est très proche de celle des essais START, cependant ici, les patientes ont toutes eu un traitement conservateur avec une radiothérapie du sein sans surimpression et aucune n'a eu d'irradiation ganglionnaire car les patientes avec atteinte ganglionnaire n'ont pas été incluses. Les résultats de cet essai n'ont pas mis en évidence de différence significative entre les deux groupes en terme de récidive locale ni de survie globale à 10 ans (6).

\section{Chapitre 2 : L'hypofractionnement dans les cancers du sein infiltrants ne majore pas la}

\section{toxicité}


L'ensemble des 4 grands essais d'hypofractionnement en radiothérapie mammaire cités ci-dessus ont rapporté des résultats de toxicité très rassurants à court et long termes. Dans l'essai START-A, des différences significatives en faveur du schéma à 39 Gy versus 50 Gy ont été rapportées avec un taux réduit d'induration mammaire de grade 2 ou 3, de télangiectasies, et d'œdème mammaire. Mais il n'a pas été montré de différence entre le schéma à 41,6 Gy et celui à 50Gy pour ces items (8). Dans ces 2 essais les taux d'ischémie cardiaque (pour les seins gauche), de fractures symptomatiques de côte et de fibrose pulmonaire symptomatique avec 10 ans de suivi ont été de l'ordre de $1 \%$ sans différence significative entre les trois schémas de fractionnement (8).

Dans l'essai START-B, moins d'effets secondaires à 10 ans ont été rapportés dans le bras hypofractionné que dans le bras contrôle. Cette réduction a été significative pour le taux de rétraction mammaire (31,2\% vs. $26,2 \%)$, de télangiectasies $(5,8 \%$ vs. $4,2 \%)$ et d'œdème mammaire (9\% vs. $5,1 \%)$. II n'a pas été observé de différence significative pour le taux d'induration mammaire (17,4\% vs. $14,3 \%)$. De façon similaire aux résultats de l'essai START A, la toxicité costale, pulmonaire et cardiaque a été minime (8).

Après analyse des patientes qui ont reçu une irradiation ganglionnaire dans les deux essais START-A et START-B, il n'a pas été montré de différence significative de toxicité liée à cette irradiation ganglionnaire entre les bras expérimentaux et le bras contrôle (18).

Dans l'essai canadien il n'a pas été retrouvé de différence significative entre les groupes pour les résultats cosmétiques tardifs $(71,3 \%$ de résultats cosmétiques bons ou excellents dans le groupe de 42,5 Gy en 16 fractions vs. 69,8\% dans le groupe de 50 Gy en 25 fractions ). 
Seulement $4 \%$ des patientes incluses dans cet essai ont eu une toxicité cutanée ou souscutanée de grade 3 (6).

Les bons résultats cliniques et la bonne tolérance montrés par ces essais permettent de recommander le traitement hypofractionné du sein seul avec un niveau de preuve IA. Les dernières recommandations de l'ASTRO proposent même d'élargir le groupe des patientes qui pourrait bénéficier de ce traitement, en incluant des patientes pré-ménopausées (sans limite d'âge), de stade (si il n'y a pas d'indication à une irradiation ganglionnaire), et en incluant des patientes qui ont reçu une chimiothérapie, tout en gardant la possibilité d'ajouter une surimpression si nécessaire (19).

Chapitre 3 : L’hypofractionnement dans les carcinomes intracanalaires

La radiothérapie mammaire, à la dose de 50 Gy en 25 fractions, réduit le risque de récidive locale à 10 ans, après chirurgie conservatrice pour un carcinome canalaire in situ, de $28 \%$ à $13 \%(20-24)$.

Suite aux résultats présentés dans les grands essais d'hypofractionnement pour les cancers du sein infiltrants, cette approche a été étudiée pour les carcinomes canalaires in situ. A ce jour, nous ne disposons que de données rétrospectives. Deux séries, une monocentrique et l'autre bi-centrique, de 266 et 220 patients respectivement, ont comparé le traitement classique de 50 Gy en 25 fractions à des schémas hypofractionnés délivrant 42,4 et 43,4 Gy en 16 fractions ou 45 Gy en 20 fractions $(25,26)$. Avec un suivi médian proche de 4 ans, ces séries n'ont pas montré de différence significative en récidive locale entre le traitement classique et 
I’hypofractionné $(25,26)$. Isfahanian et al, dans une étude de 2003 à 2008, comportant 348 patientes, avec un suivi médian de 64,8 mois, ont également retrouvé un taux de survie sans récidive locale à 5 ans similaire entre un groupe de patientes traitées par chirurgie conservatrice suivie soit d'une radiothérapie normofractionnée (50 Gy en 25 fractions) soit d'une radiothérapie hypofractionnée (40 à 42,6 Gy en 15 à 16 fractions) (94\% vs 91\% $p=0,80)(27)$.

Le plus large essai rétrospectif qui adresse ce sujet a inclus 1609 patientes entre 1994 et 2003. Cet essai a montré, en analyse multi variée, avec un suivi médian de 9,2 ans, que le schéma hypofractionné (42,4 Gy en 16 fractions) n'est pas associé à un risque accru de récidive locale en comparaison au groupe normofractionné (HR=0,8; IC95\%:0,5-1,2; $p=0,34)(28)$. A noter que le groupe normofractionné a comporté 3,5 fois moins de patientes qui ont eu une surimpression que le groupe hypofractionné.

Une méta-analyse de 4 essais (2534 patientes) a comparé les traitements normofractionnés et hypofractionnés chez des patients présentant un carcinomes canalaire in situ et n'a pas trouvé de différence significative sur le taux de récidive locale entre les deux groupes (OR: 0,78, 95\% IC: 0,58-1,03, $p=0,08)(29)$.

L'essai du Trans-Tasman Radiation Oncology Group (TROG) 07.01 est un essai de phase 3 à 4 bras qui a randomisé 1609 patientes après chirurgie conservatrice d'un carcinome canalaire in situ entre une radiothérapie hypofractionnée en 3 semaines ou un traitement normofractionné avec ou sans boost (30). Cet essai est fermé aux inclusions depuis 2014 et les 
résultats sont très attendus pour répondre définitivement à cette question avec données prospectives robustes (30).

\section{Chapitre 4 : Qu'en est-il de la radiothérapie hypofractionnée post mastectomie et/ou en cas}

\section{d'irradiation ganglionnaire?}

Bien que la radiosensibilité de la peau, de la paroi thoracique ou des organes à risque (OAR) de voisinage soit la même après traitement conservateur ou après mastectomie radicale il existe souvent une hésitation à traiter en radiothérapie hypofractionnée les parois et/ou les aires ganglionnaires. Ceci est très vraisemblablement lié d'une part aux doses souvent plus importantes délivrées aux OAR en cas d'irradiation pariétale et ganglionnaire et d'autre part au faible niveau de preuve associé à l'utilisation de la radiothérapie hypofractionnée dans ces situations. Toutefois, 513 des 5861 patients des essais START pilot, START A et START B ont eu une mastectomie radicale. Bien que ces essais n'aient pas été spécifiquement construits pour analyser ce sous-groupe et qu'ils n'aient pas la puissance statistique pour conclure sur cette sous population, il est intéressant de noter qu'avec 5 et 10 ans de suivi aucune crainte particulière n’a été identifié concernant le risque de toxicité ou l'efficacité lié à la radiothérapie hypofractionnée post-mastectomie (8,31-33).

Dans les essais START, seuls 470 des 5861 patients ont reçu une irradiation ganglionnaire. Ceci représente respectivement $20,6 \%, 14,2 \%$ et $7,3 \%$ des essais START pilot, START A et START B. Ces irradiations ganglionnaires ont concerné principalement les niveaux sus claviculaires (niveau IV) et sous claviculaire (niveau III), rarement les niveaux I et II dont la 
partie basse à toute fois pu être traitée par les faisceaux tangentiels. Là encore, une analyse rétrospective, sans la puissance statistique suffisante pour conclure de manière ferme, a été réalisée. Celle-ci ne retrouve aucun élément en faveur d'une majoration de la toxicité (en particulier concernant la mobilité de l'épaule ou le risque de lymphoedème) ni d'une moindre efficacité dans le bras hypo versus normofractionné (8,31-33). Par ailleurs, aucun cas de plexite brachiale n'a été rapporté dans le bras hypofractionné de l'essai START B avec 10 ans de suivi (8). Seul un cas de plexite brachiale a été rapporté à 10 ans dans l'essai START A. Celui-ci est survenu dans le bras délivrant 41,6Gy en 13 fractions sur 5 semaines (8). Ainsi les auteurs concluent à un taux observé très faible de plexite dont le risque serait même statistiquement plus faible en cas de radiothérapie hypofractionnée si l’on en croit la modélisation des risques d'effets secondaires proposée par Yarnold basée sur un $\alpha / \beta$ de 1,5 Gy (31).

C'est sur la base de ces résultats et de l'expérience de nombreux centres britanniques et hollandais utilisant la radiothérapie hypofractionnée, hors protocole, avec plus de 10 ans de recul, que les recommandations du royaume unis précisent depuis 2016 qu'il n'y a pas d'indication à utiliser plus de 15 fractions pour une radiothérapie du sein, de la paroi ou des aires ganglionnaires (34). Le schéma hypofractionné délivrant 40Gy en 15 fractions est donc le standard de traitement pour toute radiothérapie adjuvante locale ou locorégionale pour un cancer du sein au royaume uni (34).

Toutefois aux états unis, considérant ces éléments comme un niveau de preuve faible, Khan et al ont plus récemment, montré dans un essai de phase II, portant sur 69 patients traités par radiothérapie hypofractionnée après mastectomie, un profil de tolérance et d'efficacité tout à fait rassurant (35). Dans cet essai, dont le suivi médian est de 32 mois seulement, le 
schéma hypofractionné utilisé a délivré 36,63 Gy en 11 fractions sur 11 jours sur la paroi et les aires ganglionnaires de drainage comportant les niveaux III et IV +/- I et II. Le traitement de la chaîne mammaire interne a été laissé à la discrétion des investigateurs. Ces données et celles de la littérature ont permis aux auteurs de dessiner l'essai de phase III Alliance A221505, qui vient d'ouvrir en février 2018, et a pour objectif de comparer sur 880 patientes, la radiothérapie normo versus hypofractionnée post mastectomie chez des patientes avec reconstruction mammaire. Une enquête de pratique aux états unis a montré récemment que sur 113081 patientes traitées pour un cancer du sein entre 2004 et 2014 seules 1,1\% ont reçu une radiothérapie hypofractionnée après mastectomie. La principale raison évoquée par les auteurs est le niveau de preuve faible. II existe tout de même une augmentation significative de I'utilisation des schémas hypofractionnés après mastectomie sur les dernières années (36).

En Europe, deux essais de phase III testent actuellement le schéma hypofractionné délivrant 40Gy en 15 fractions versus le schéma normofractionné classique afin d'établir enfin, avec un niveau de preuve IA, la non infériorité ou non de la radiothérapie hypofractionnée locorégionale adjuvante dans les cancers du sein traités de manière conservatrice ou non, avec ou sans reconstruction, quel que soit l'âge (> 18 ans). Il s'agit d'une part de l'essai international du groupe coopératif danois DBCG HYPOIl et de l'essai Français HYPOG-01. Avec plus de 1000 patients prévus dans chaque essai, dont plus de la moitié déjà recrutés dans chacun d'eux, l'objectif principal est d'évaluer la toxicité tardive du schéma hypo versus normofractionné. Une méta-analyse prospective des deux essais est prévue dans le cadre du projet Skagen I qui réunit ces deux essais offrant ainsi une occasion unique de faire changer les pratiques basée sur un haut niveau de preuve (ClinicalTrials.gov Identifier: NCT02384733 et NCT03127995). 


\section{Chapitre 5 : jusqu'où aller dans l'hypofractionnement en sénologie?}

Le rapport $\alpha / \beta$ caractérise la sensibilité des tissus à la dose par fraction. Un rapport $\alpha / \beta$ de 4 Gy pour les adénocarcinomes mammaires est communément retrouvé dans la littérature (37). Une estimation récente de ce ratio, basée sur les données des essais START pilot et START A donne un ratio $\alpha / \beta$ de 4,6 Gy (IC $95 \%: 1,1-8,1)(33,38)$. Ce ratio est confirmé par les données des essais canadiens et START B délivrant des doses par fraction de 2,67 Gy $(33,38)$. Dans ces essais, l'augmentation de la dose par fraction n'a pas été associée à une augmentation de la toxicité en particulier à long terme. Selon le modèle linéaire quadratique, avec un $\alpha / \beta$ de 3 et 4 Gy, cinq fractions de 5,7 et 6 Gy seraient respectivement équivalentes à 25 fractions de 2 Gy (39). Courdi et al ont rapporté une série de 115 patients, âgées en moyenne de 82,7 ans, traitées par radiothérapie et hormonothérapie exclusive pour un cancer du sein non métastatique à raison d'une fraction de 6,5 Gy par semaine sur cinq semaines sur l'ensemble du sein avec une surimpression délivrée en 1 à 3 fractions hebdomadaire de 6,5 Gy (40). Avec un suivi médian de 41 mois, 46 patients ont présenté des effets secondaires tardifs. L'effet secondaire tardif le plus fréquent est l'induration du sein en rapport avec une fibrose sous cutanée retrouvée chez 19 patientes avec un grade 1, chez 21 patientes avec un grade 2 et chez 6 patientes avec un grade 3 (40). Une autre étude rétrospective française, rapportée par Kirova et al, chez 50 patients traités par radiothérapie hypofractionnée adjuvante, délivrant 32,5 Gy en 5 fractions de 6,5 Gy, sur 5 semaines, sur l'ensemble du sein, a également montré une bonne faisabilité et une toxicité limitée. En effet, avec un suivi médian de 93 mois (9-140 mois), une fibrose du sein de grades 1 et 2 a été rapportée chez $33 \%$ des patients. II n’a pas été 
constaté de séquelle à long terme chez $67,3 \%$ des patients. Aucune toxicité de grades 3 ou 4 n'a été rapportée (41). Afin de valider prospectivement et de manière randomisée ces données concernant la radiothérapie hypofractionnée avec des doses par fraction voisines des 6 Gy, le " FAST trialists group » anglais a réalisé l'essai FAST. Cet essai de phase III a comparé 3 bras de traitement : I'un, délivrant 50 Gy en 25 fractions de 2Gy par jour sur 5 semaines, les autres délivrant respectivement 5 fractions de 5,7 Gy ou 6 Gy par semaine sur 5 semaines sur l'ensemble du sein, sans surimpression, après chirurgie pour un cancer du sein de stade précoce. L'étalement dans les 3 bras a été le même pour pouvoir juger spécifiquement de l'influence de la dose par fraction. D'octobre 2004 à Mars 2007, 915 patientes, de 18 centres anglais, ont été randomisées. Avec un suivi médian de 37,3 mois et un peu plus de 300 patientes par bras, le risque relatif de modification légère ou marquée du sein rapporté est de 1,7 (IC 95\% 1,26-2,29, p<0,001) pour le bras délivrant une dose totale de 30 Gy et de 1,15 (IC $95 \%$ 0,82-1,60, $p=0,489$ ) pour le bras délivrant une dose totale de 28,5 Gy en comparaison avec le bras standard délivrant une dose totale de 50 Gy. A 3 ans, cet essai montre que le bras délivrant 28,5 Gy en 5 fractions est comparable au bras délivrant 50 Gy en 25 fractions et qu'il engendre une toxicité moindre que celle du bras délivrant 30 Gy en 5 fractions (38). Les résultats avec un suivi à plus long terme de cet essai FAST sont en attente. Dans l'intervalle, ce même groupe a lancé une étude pilote sur 30 patientes traitées à la dose totale de 30 Gy en 5 fractions sur 15 jours, sur l'ensemble du sein, après chirurgie conservatrice, afin d'évaluer la faisabilité d'une accélération de ce schéma hypofractionné. Les premiers résultats publiés par Martin et al ont montré une bonne faisabilité et une toxicité acceptable sans toxicité de grade supérieur à 2 en aigue et aucune modification marquée ou sévère du sein à 2 ans du traitement 
(42). A la suite de ces résultats ce même « FAST trialists group » a lancé l'essai FAST-Forward qui pose la question d'accélérer même d'avantage ce schéma hypofractionné. Cet essai FASTForward est un essai de phase III randomisé, multicentrique qui compare 3 schémas de radiothérapie adjuvante sur l'ensemble du sein après chirurgie conservatrice ou mastectomie +/- reconstruction mammaire: un bras standard délivrant 40 Gy en 15 fractions sur 3 semaines et deux bras hypofractionnés accélérés délivrant 27 Gy en 5 fractions sur 1 semaine pour l'un et 26 Gy en 5 fractions sur 1 semaine pour l'autre. Cet essai, ouvert en 2015, prévoit d'inclure au royaume unis 4000 patientes et a pour objectif principal le contrôle local (ISRCTN 19906132).

\section{Conclusion}

Les traitements adjuvants hypofractionnés modérés sont devenus le traitement standard chez les patientes âgées avec un cancer du sein de stade précoce, après chirurgie conservatrice. Ils permettent d'améliorer l'adhésion aux traitements, et d'assurer des taux de contrôle local non-inférieurs au traitement classique avec un profil de toxicité superposable. Néanmoins, leur place dans les carcinomes in situ et l'irradiation ganglionnaire reste à confirmer pour atteindre un niveau de preuve I. Les limites de I'hypofractionnement optimal sont encore en cours d'évaluation. Les données des essais en cours élargiront très vraisemblablement les indications de ces schémas de traitement, en augmentant ainsi le nombre de patientes qui pourraient bénéficier en toute sécurité de traitement plus courts.

Les auteurs déclarent ne pas avoir de conflits d'intérêt. 


\section{Références}

1. McGale P, Taylor C, Correa C, Cutter D, Duane F, Ewertz M, et al. Effect of radiotherapy after mastectomy and axillary surgery on 10-year recurrence and 20-year breast cancer mortality: Meta-analysis of individual patient data for 8135 women in 22 randomised trials. Lancet. 2014;383:2127-35.

2. Darby S, McGale P, Correa C, Taylor C, Arriagada R, Clarke M, et al. Effect of radiotherapy after breast-conserving surgery on 10-year recurrence and 15-year breast cancer death: Meta-analysis of individual patient data for 10801 women in 17 randomised trials. Lancet. 2011;378:1707-16.

3. Clarke M, Collins R, Darby S, Davies C, Elphinstone P, Evans V, et al. Effects of radiotherapy and of differences in the extent of surgery for early breast cancer on local recurrence and 15-year survival: an overview of the randomised trials. Lancet. 2005;(366):2087-106.

4. Bartelink H, Maingon P, Poortmans P, Weltens C, Fourquet A, Jager J, et al. Whole-breast irradiation with or without a boost for patients treated with breast-conserving surgery for early breast cancer: 20-year follow-up of a randomised phase 3 trial. Lancet Oncol. 2015;16(16):47-56.

5. Athas WF, Adams-Cameron M, Hunt WC, Amir-Fazli A, Key CR. Travel Distance to Radiation Therapy and Receipt of Radiotherapy Following Breast-Conserving Surgery. J Natl Cancer Inst. 2000;92(3):269-71. 
6. Whelan TJ, Pignol J-P, Levine MN, Julian JA, Mackenzie R, Parpia S, et al. Long-Term Results of Hypofractionated Radiation Therapy for Breast Cancer. N Engl J Med. 2010;362:513-20.

7. Owen JR, Ashton A, Bliss JM, Homewood J, Harper C, Hanson J, et al. Effect of radiotherapy fraction size on tumour control in patients with early-stage breast cancer after local tumour excision: long-term results of a randomised trial. Lancet Oncol. 2006;7:467-71.

8. Haviland JS, Owen JR, Dewar JA, Agrawal RK, Barrett J, Barrett-Lee PJ, et al. The UK Standardisation of Breast Radiotherapy (START) trials of radiotherapy hypofractionation for treatment of early breast cancer: 10-year follow-up results of two randomised controlled trials. Lancet Oncol. 2013;14:1086-94.

9. Smith BD, Bentzen SM, Correa CR, Hahn CA, Hardenbergh PH, Ibbott GS, et al. Fractionation for whole breast irradiation: an american society for radiation oncology (ASTRO) evidence-based guideline. Int J Radiat Oncol Biol Phys. 2011;81(1):59-68.

10. Institut national du cancer. Recommandations professionelles. Cancer du sein infiltrant non métastatique. Questions d'actualité. Rappport intégral. Coll. Avis. 2012.

11. NCCN Clinical Practice Guidelines in Oncology. (NCCN Guidelines ${ }^{\circledR}$ ). Breast Cancer. Fort Washington, PA: National Comprehensive Cancer Network; 2015.

12. Hennequin C, Barillot I, Azria D, Belkacémi Y, Bollet M, Chauvet B, et al. Radiothérapie du cancer du sein. Cancer/Radiotherapie. 2016;20:S139-46. 
13. Oncologica. Al di R. La Radioterapia dei Tumori della Mammella Indicazioni e Criteri Guida. [Internet]. Available from: http://vecchio.radioterapiaitalia.it/ allegato_1222_796.phtml

14. Sedlmayer F, Sautter-Bihl M-L, Budach W. DEGRO practical guidelines: radiotherapy of breast cancer I: radiotherapy following breast conserving therapy for invasive breast cancer. Strahlenther Onkol. 2013;189:825-33.

15. Gay HA, Barthold HJ, O’Meara E et al. Female Pelvis Normal Tissue RTOG Consensus Contouring Guidelines. [Internet]. [cited 2016 Dec 9]. Available from: https://www.rtog.org/LinkClick.aspx?fileticket=P5eAjYB900w\%3D\&tabid=355

16. NBOCC. A systematic literature review of hypofractionated radiotherapy for the treatment of early breast cancer. In: Cancer Australia. [11]e. Surry Hills, NSW; 2010.

17. Harnett A, Smallwood J, Titshall V, Champion A. Diagnosis and treatment of early breast cancer, including locally advanced disease - summary of NICE guidance. Br Med J. 2009;(338):b438.

18. Haviland JS, Mannino M, Griffin C, Porta N, Sydenham M, Bliss JM, et al. Phase III randomised trial Late normal tissue effects in the arm and shoulder following lymphatic radiotherapy: Results from the UK START (Standardisation of Breast Radiotherapy) trials. Radiother Oncol. 2018;126:155-62.

19. Smith BD, Bellon JR, Blitzblau R, Freedman G, Haffty B, Hahn C, et al. Radiation therapy for the whole breast: Executive summary of an American Society for Radiation Oncology 
(ASTRO) evidence-based guideline. Pr Radiat Oncol. 2018;8(3):145-52.

20. Houghton J, George W, Cuzick J. Radiotherapy and tamoxifen in women with completely excised ductal carcinoma in situ of the breast in the UK, Australia, and New Zealand: randomised controlled trial UK. Lancet. 2003;(362):95-102.

21. Fisher B, Dignam JJ, Wolmark N, Mamounas EP, Costantino JP, Poller W, et al. Lumpectomy and radiation therapy for the treatment of intraductal breast cancer: findings from National Surgical Adjuvant Breast and Bowel Project B-17. J Clin Oncol. 1998;16(2):441-52.

22. Julien J-P, Bijker N, Fentiman IS, Peterse JL, Delledonne V, Rouanet $\mathrm{P}$, et al. Radiotherapy in breast-conserving treatment for ductal carcinoma in situ: first results of the EORTC randomised phase III trial 10853. Lancet. 2000;355:528-33.

23. Donker M, Litière S, Werutsky G, Julien JP, Fentiman IS, Agresti R, et al. Breast-conserving treatment with or without radiotherapy in ductal carcinoma in situ: 15-year recurrence rates and outcome after a recurrence, from the EORTC 10853 randomized phase III trial. J Clin Oncol. 2013;31(32):4054-9.

24. Correa C, McGale P, Taylor C, Wang Y, Clarke M, Davies C, et al. Overview of the randomized trials of radiotherapy in ductal carcinoma in situ of the breast. J Natl Cancer Inst - Monogr. 2010;41:162-77.

25. Williamson D, Dinniwell R, Fung S, Pintilie M, Done SJ, Fyles AW. Ductal carcinoma in situ Local control with conventional and hypofractionated adjuvant radiotherapy after 
breast-conserving surgery for ductal carcinoma in-situ. Radiother Oncol. 2010;95:31720.

26. Wong P, Lambert C, Agnihotram R V, David M, Duclos M, Freeman CR. Ductal carcinoma in situ - the influence of the radiotherapy boost on local control. Int J Radiat Oncol Biol Phys. 2012;82(2):e153-8.

27. Isfahanian N, Al-Hajri T, Marginean H, Chang L, Caudrelier J-M. Hypofractionation Is an Acceptable Alternative to Conventional Fractionation in the Treatment of Postlumpectomy Ductal Carcinoma In Situ With Radiotherapy. Clin Breast Cancer. 2017;17(2):77-85.

28. Lalani N, Paszat L, Sutradhar R, Thiruchelvam D, Nofech-Mozes S, Hanna W, et al. Longterm Outcomes of Hypofractionation Versus Conventional Radiation Therapy After Breast-Conserving Surgery for Ductal Carcinoma In Situ of the Breast. Int J Radiat Oncol Biol Phys. 2014;90(5):1017-24.

29. Nilsson C, Valachis A. The role of boost and hypofractionation as adjuvant radiotherapy in patients with DCIS: A meta-analysis of observational studies. Radiother Oncol. $2015 ; 114: 50-5$.

30. TROG (Trans Tasman Radiation Oncology Group) Cancer Research. TROG 07.01. A randomized phase III study of radiation doses and fractionation schedules in non-low risk ductal carcinoma in situ (DCIS) of the breast. [Internet]. [cited 2018 Jun 20]. Available from: https://clinicaltrials.gov/ct2/show/NCT00470236 
31. Yarnold J. changes in radiotherapy fractionation-breast cancer. Br J Radiol. 2018;91(20170849).

32. Hopwood P, Haviland JS, Sumo G, Mills J, Bliss JM, Yarnold JR. Comparison of patientreported breast, arm, and shoulder symptoms and body image after radiotherapy for early breast cancer: 5-year follow-up in the randomised Standardisation of Breast Radiotherapy (START) trials. Lancet Oncol. 2010;11:231-40.

33. Bentzen SM, Agrawal RK, Aird EGA, Barrett JM, Barrett-Lee PJ, Bliss JM, et al. The UK Standardisation of Breast Radiotherapy (START) Trial A of radiotherapy hypofractionation for treatment of early breast cancer: a randomised trial. Lancet Oncol. 2008;9:331-41.

34. Royal College of Radiologists. Consensus statement on hypofractionation in breast cancer. 2016.

35. Khan AJ, Poppe MM, Goyal S, Kokeny KE, Kearney T, Kirstein L, et al. Hypofractionated postmastectomy radiation therapy is safe and effective: First Results from a prospective phase II trial. J Clin Oncol. 2017;35(18):2037-43.

36. Venigalla S, Guttmann DM, Jain V, Sharma S, Freedman GM, Shabason JE. Trends and Patterns of Utilization of Hypofractionated Postmastectomy Radiotherapy: A National Cancer Database Analysis. Clin Breast Cancer. 2018;in press.

37. Hennequin C, Dubray B. Le rapport alpha/bêta revisité à l'heure de l'hypofractionnement Alpha/beta ratio revisited in the era of hypofractionation. Cancer / Radiother. $2013 ; 17: 344-8$. 
38. FAST Trialists group T. First results of the randomised UK FAST Trial of radiotherapy hypofractionation for treatment of early breast cancer (CRUKE/04/015). Radiother Oncol. 2011;100:93-100.

39. Jones B, Dale RG, Deehan C, Hopkins KI, Morgan DAL. The Role of Biologically Effective Dose (BED) in Clinical Oncology. Clin Oncol. 2001;13:71-81.

40. Courdi A, Ortholan C, Hannoun-Lévi J-M, Ferrero J-M, Largillier R, Balu-Maestro C, et al. Breast cancer in elderly Long-term results of hypofractionated radiotherapy and hormonal therapy without surgery for breast cancer in elderly patients. Radiother Oncol. $2006 ; 79(2): 156-61$.

41. Kirova YM, Campana F, Savignoni A, Laki F, Muresan M, Dendale R, et al. Breastconserving treatment in the elderly: long-term results of adjuvant hypofractionated and normofractionated radiotherapy. Int J Radiat Oncol Biol Phys. 2009;75(1):76-81.

42. Martin S, Mannino M, Rostom A, Tait D, Donovany E, Eagle S, et al. Acute Toxicity and 2year Adverse Effects of $30 \mathrm{~Gy}$ in Five Fractions over 15 Days to Whole Breast after Local Excision of Early Breast Cancer. Clin Oncol. 2008;20:502-5. 